\title{
Systematic numerical investigation of the role of hierarchy in heterogeneous bio- inspired materials
}

\author{
Federico Bosia ${ }^{1}$, Federico Della Croce ${ }^{2}$ and Nicola M. Pugno ${ }^{3,4,5^{*}}$ \\ ${ }^{1}$ Department of Physics and "Nanostructured Interfaces and Surfaces" Centre of Excellence, \\ University of Torino, 10125, Torino, Italy. \\ ${ }^{2}$ Department of Control and Computer Engineering, Politecnico di Torino, 10129, Torino, Italy. \\ ${ }^{3}$ Laboratory of Bio-Inspired Nanomechanics "Giuseppe Maria Pugno", Department of Structural, \\ Geotechnical and Building Engineering, Politecnico di Torino, 10129, Torino, Italy. \\ ${ }^{4}$ National Institute of Nuclear Physics, National Laboratories of Frascati, Via E. Fermi 40, 00044, \\ Frascati, Italy. \\ ${ }^{5}$ National Institute of Metrological Research, Strada delle Cacce 91, I-10135, Torino, Italy.
}

*Corresponding author: nicola.pugno@polito.it

\section{Paper submitted to Special Issue of JMBBM}

""Science and engineering of natural materials: Merging structure and material" 

Abstract:
It is well known that hierarchical structure is an important feature in biological materials to optimize various properties, including mechanical ones. It is however still unclear how these hierarchical architectures can improve material characteristics, for example strength. Also, the transposition of these structures from natural to artificial bioinspired materials remains to be perfected. In this paper, we introduce a numerical method to evaluate the strength of fibre-based heterogeneous biological materials and systematically investigate the role of hierarchy. Results show that hierarchy indeed plays an important role and that it is possible to "tune" the strength of bio-inspired materials in a wide range of values, in some cases improving the strength of non hierarchical structures considerably. 


\section{Introduction}

It is known that many biological materials and organisms display fascinating physical and mechanical properties, which have up to now been hard to replicate in artificial materials and systems. One of these is the ability to combine exceptional strength and toughness, which occurs for example in nacre, bone and dentine (Espinosa et al., 2011; Pugno, 2006; Wegst and Ashby, 2004), or the smart adhesion which is found in spiders and geckos (Autumn et al., 2000; Autumn and Peattie, 2002; Foelix, 1996; Tian et al., 2006). An important feature underlying these properties is thought to be material structure and hierarchy (Fratzl and Weinkamer, 2007; Lakes, 1993). A prime example of this is spider silk, whose hierarchical structure ranges from nanostructure to macrostructure and consists of an amorphous network of chains and $\beta$-sheet crystals constituted by poly-(Gly-Ala) and poly-Ala domains (Ackbarow et al., 2007; Keten et al., 2010). Molecular dynamics and atomistic simulations have shown how the specific structure and bonding at molecular level affects macroscopic properties like strength and toughness (Bratzel and Buehler, 2012; Keten et al., 2010; Nova et al., 2010).

Aside from spider silk, a great number of biological materials are inherently structurally hierarchical. The hierarchical structure of tendon, taken from (Riley, 2005 ) is shown in Fig1a. Another example is the case of bone, where variability at the nanometer level lies in the shape and size of mineral particles, at the micron level in the arrangement of mineralized collagen fibers into lamellar structures, and beyond in the inner architecture, the porosity and the shape of the bone. Various studies show the dependence of the mechanical properties of bone on all these parameters (Currey, 2002; Gibson et al., 1995; Launey et al., 2010; Rho et al., 1998; Weiner and Wagner, 1998). Other biological systems that have been studied to assess the role of hierarchy are tendons (Puxkandl et al., 2002), protein materials (Gao, 2006), Gecko adhesion (Yao and Gao, 2006), tissue 
growth (Cranford and Buehler, 2011).

Given a hierarchical organization, various designs are possible, by altering the type and arrangement of the components at different levels. Hierarchy and functional grading frequently lead to variable mechanical properties at different length scales, i.e. overall mechanical properties are often quite different from those of constituents (Lakes, 1993; Pugno, 2006), and many natural materials can be considered an equivalent of artificial composite materials. For example, stiff biological materials are often composites with the smallest components mostly in the nanometer range (Gao and $\mathrm{Ji}, 2004)$. In the case of plants or insect cuticles, a polymeric matrix is reinforced by stiff polymer fibers, such as cellulose or keratin (Vincent, 1999), and in the case of bone or dentin even stiffer structures are obtained using a fibrous polymeric matrix reinforced by hard carbonated hydroxylapatite particles (Currey, 1999).

One possible hypothesis is therefore that the exceptional mechanical behavior of biological materials is due to two essential elements: hierarchy and material heterogeneity. To verify this conjecture, a number of theoretical models which include both these elements have been formulated, including molecular dynamics or atomistic simulations (Buehler et al., 2009; Currey, 1999, 2003; Gao and Ji, 2004; Pugno, 2006). A simplified numerical approach is the fiber bundle model (FBM) which has been extensively studied during the past years (Pradhan et al., 2010). This model consists of a set of parallel fibers having statistically distributed strengths. The sample is loaded parallel to the fiber direction, and the fibers fail if the load exceeds their threshold value, with the load carried by the broken fiber being redistributed among the intact ones. The Equal Load Sharing (ELS) formulation is most often adopted, whereby after each fiber break the stress is equally distributed on the intact fibers, neglecting stress concentrations in the vicinity of failed regions. Based on this model, we developed a hierarchical formulation of the FBM ("HFBM") and 
used it to calculate the space elevator cable strength including the role of defects (Pugno et al.,

2008). With this model we also studied the strength and toughness of nanotube-based composites, starting from the properties and volume fractions of the fragile and ductile constituents (Bosia et al., 2010). In recent work, we addressed the issue of the synergy between hierarchy and material mixing to enhance the mechanical performance of composites, finding evidence that some hierarchical configurations lead to an improvement with respect to the non hierarchical case (Bosia et al., 2012) An important numerical study of damage evolution in hierarchical FBMs was also recently carried out by Mishnaevsky (Mishnaevsky, 2011).

However, despite the recent advances in this field, a systematic study addressing the role of pure hierarchy (independently of the specific material system it refers to), its interaction with material heterogeneity, and their effect on macroscopic mechanical properties is still missing. In this paper, we therefore wish to begin such a systematic study, and investigate the possibility of tuning and optimizing the strength of hierarchical fibre bundles composed of different fibre types as a function of hierarchy and distribution of different fibre types.

The paper is structured as follows: in Section 2, we introduce the numerical model used to calculate the strength of hierarchical fiber bundle architectures in composite materials and the evaluation procedure; in Section 3, we present results of calculations and their discussion; finally, conclusions and outlook are given.

\section{Hierarchical Fibre Bundle Model}

\subsection{Model implementation}

As mentioned above, a Hierarchical Fibre Bundle Model (HFBM) was adopted for simulations. The model used here is related to that proposed by Bosia et al. (Bosia et al., 2008) and Pugno et al. (Pugno et al., 2008). As with all FBMs, the individual fibres have randomly-assigned statistically- 
distributed strengths, in our case according to the 2 parameter Weibull distribution (see Fig.2), which is described by the following equation (Weibull, 1939, 1951):

$$
P(\sigma)=1-e^{-\left(\frac{\sigma}{\sigma_{0}}\right)^{m}}
$$

The model is based on an Equal-Load-Sharing (ELS) FBM approach, replicated in a hierarchical scheme at various length scales ("levels") to predict from statistical considerations the mechanical behaviour of different hierarchical architectures. Also, in order to model heterogeneous fibrous media, the fibres of each bundle can assume different mechanical properties. The $k$-th fibre type is characterized by a Young's moulus $E_{k}$, length $l_{k}$, cross-sectional area $A_{k}$, and Weibull-distributed fracture strengths, the latter characterized by a scale parameter $\sigma_{0 k}$ and shape parameter $m_{k}$. The various types of fibres combine in forming bundles, with complex mechanical behaviour emerging from the mechanical properties and arrangement of the constituent fibres. The specimen's stressstrain behaviour is determined by imposing an increasing displacement and "rupturing" individual fibres in the bundle (i.e. setting their stiffness to zero) when their statistically assigned strength is exceeded. After each fracture event, the load is redistributed uniformly among the fibres in the same bundle as the fractured one (ELS). The bundle strength strength is obtained as the maximum stress value reached in the simulation before failure, i.e. when all parallel fibres of the bundle have failed. Since the fibre strengths are assigned randomly according to the Weibull distribution, results differ for each simulation, and average trends can be derived from repeated simulations.

Hierarchy is implemented as described by Pugno et al. (Pugno et al., 2008) and Bosia et al. (Bosia et al., 2008), schematically illustrated in Fig.3, i.e. the input mechanical behaviour of a level $i=h-1$ "fibre" or subvolume is statistically inferred from the output deriving from hundreds of level 
$h$ simulations, that of a level $i=h-2$ subvolume from level $i=h-1$ simulations, and so on, down to the

lowest hierarchical level $i=1$. Overall, the specimen is modelled as an ensemble of $N_{l}$ subvolumes arranged in a bundle. Each of these subvolumes is in turn constituted by $N_{2}$ subvolumes, arranged in a bundle as before. This scheme is applied for $h$ "generations", up to a level $h$ subvolume, which is constituted $N_{a}$ type " $a$ " fibres, $N_{b}$ type " $b$ " fibres, and so on.

Since at every single fibre failure the load is only redistributed among parallel fibres in the "local" bundle, fibre failures in different bundles "interact" only at the next hierarchical level. Thus, comparing hierarchical bundles with the same overall number of fibres and fibre-type percentages, but different hierarchical architectures, amounts to considering different stress redistribution schemes in the material. To simplify the problem, we initially consider only 2 fibre types ( $a$ and $b$, basically a "matrix" and a "reinforcement" as in composites) and symmetrical structures (each bundle is split into identical bundles at each hierarchical level). Thus, to define the overall bundle, we require the following parameters:

\section{i. $\quad$ Fixed parameters:}

$N$ (integer): total number of fibres

$E_{a}$ and $E_{b}$ (real number): stiffnesses for fibres $a$ and $b$

$\sigma_{a}$ and $\sigma_{b}$ (real number): Weibull scale parameters for fibres $a$ and $b$

$m_{a}$ and $m_{b}$ (real number): Weibull shape parameters for fibres $a$ and $b$

$\alpha$ (real number): Fraction of type $a$ fibres, so that $(1-\alpha)$ is the fraction of type $b$ fibres. $0<\alpha<1$

\section{ii. Variable parameters:}

$h$ (integer): number of hierarchical levels

$n_{1}, n_{2} \ldots n_{h-1}$ (integers): number of parallel bundles at hierarchical levels $i=1,2 \ldots(h-1)$

$n_{a}$ (integer): number of parallel bundles of type $a$ fibres at the last hierarchical level $i=h$ 
$n_{b}$ (integer): number of parallel bundles of type $b$ fibres at the last hierarchical level $i=h$

Clearly, for $h>1$, any configuration with $n_{i}=1$ corresponds to a (h-1) architecture, e.g. a $h=3$ configuration with $n_{1}=1, n_{2}=2, n_{a}=5, n_{b}=10$ is identical to $h=2, n_{l}=2, n_{a}=5, n_{b}=10$. Therefore, for $h>1$, only configurations with $n_{i}>1$ are considered.

The number of free parameters depends on the number of hierarchical levels of the considered structures. Two equivalent parameters are the number of type $a$ and $b$ fibres, respectively, in each bundle at level $h$, i.e. $N_{a}=\frac{\alpha N}{n_{1} n_{2} \ldots n_{i-1} n_{a}}$ and $N_{b}=\frac{(1-\alpha) N}{n_{1} n_{2} \ldots n_{i-1} n_{b}}$. The only constraints on the variable parameters are thus that $N_{a}$ and $N_{b}$ must be integers. To be able to satisfy this constraint, $N$ needs to be sufficiently large, however this increases computational time, so a compromise is required (typically $N=10^{2} \div 10^{4}$ ). To avoid excessively time-consuming problems, we can consider initially $h<4$. Each simulation is repeated typically $10^{2}$ times to obtain a mean strength for the considered structure.

To illustrate the procedure, let us consider an example of a 3-level hierarchical structure ( $h=3$ ) with $N=3600$ and $\alpha=0.2$. The chosen mechanical parameters are $E_{a}=1000 \mathrm{GPa}$ and $E_{b}=10 \mathrm{GPa}$ for the Young's moduli, and $\sigma_{a}=100 \mathrm{GPa} \sigma_{b}=1 \mathrm{GPa}, m_{a}=2, m_{b}=3$ for the Weibull scale and shape parameters, respectively. One possible structure with these parameters is shown in Fig.3a. Each "box" in the figure represents a fibre bundle. $N_{i}$ indicates the number of fibres in each bundle at hierarchical level $i$. A fibre bundle at hierarchical level $i$ is equivalent to a single fibre at hierarchical level $i-1$. The distinction between fibres $a$ and $b$ occurs only at the highest hierarchical level $i=h$. We observe here that a rule of mixtures (Gibson, 2007) for this non-hierarchical configuration yields a strength value of $18.44 \mathrm{GPa}$. This is because the rule of mixtures is strictly be valid only in the case of simultaneous failure of all fibres in the bundle, which does not occur because of the statistical distribution of the fibre strengths. Typically, in this study the non-hierarchical bundle reaches its 
maximum stress (i.e. strength value) when about $50 \%$ of its constituent fibres (both $a$ and $b$ ) have

failed, leading to the cited strength value of about $9.1 \mathrm{GPa}$. Consequently, the rule of mixtures is only an upper bound for the mean strength, and does not provide a reliable estimation method in this case.

The strength of hierarchical structures with the same fixed parameters (specified above) is thus compared to the non-hierarchical $(h=0)$ fibre bundle (Fig.3b), the "default configuration", where all fibres are in parallel $\left(N=3600, N_{a}=720, N_{b}=2880\right)$. The latter configuration has a mean strength of 9.1 GPa. In order to evaluate the variation of bundle strength with hierarchy level and type, all possible configurations are systematically considered for $h=1,2$ and 3 . The number of possible configurations for the given parameters are 1260 for $h=1,4690$ for $h=2$, and 8641 for $h=3$, respectively. These numbers are calculated numerically for by determining all $n_{1}$ (and $n_{2}$ if $h=3$ ), $n_{a}$ and $n_{b}$ values that give rise to integer values for $N_{a}$ and $N_{b}$. Since single-fibre strengths are randomly assigned based on a Weibull distribution, there is some variability in results for each given configuration. Thus, simulations are repeated $10^{2}$ times for each configuration and a mean value and standard deviation are calculated.

\subsection{Hierarchical configuration analysis}

Regarding the number of possible configurations for given $N$ and $\alpha$ parameters, some general considerations can be made. Given an even number $N$ of springs, if we denote by $\hat{h}$ the maximum number of levels allowed for $N$, this number is at most the total number of prime factors (distinct or not) of $N$. On the other hand, the total number $T(N)$ of different configurations must take into account the number of levels $l=2 \ldots . \hat{h}$ and for each level $l$ all related configurations. As explained above, at least two fibres must be present in each bundle at every level, or else the configuration 
must be considered as belonging to the lower hierarchical level. $T(N)$ is significantly larger than $N$

already for a limited value of $\hat{h}$. Indeed, if we consider the case where $N$ is a power of 2 (namely $\left.N=2^{h}\right)$ and $\alpha=0.5$, then we have $\hat{h}$ prime factors all equal to 2 and $\alpha N=(1-\alpha) N=2^{h-1}$. Hence, as $N_{a}$ and $N_{b}$ must be integers, we have a feasible configuration if the product $n_{1} \cdot n_{2} \cdot \ldots\left(n_{l}-1\right)=2^{j}$ for any integer $j=1, \ldots, \hat{h}-1$, where $l=1, \ldots, j$ denotes the number of levels of this configuration. Notice that, as $n_{l} \cdot n_{2} \cdot \ldots\left(n_{l}-1\right)$ are all integers, they are also a power of 2 . Correspondingly, the total number of different combinations such that $n_{l} \cdot n_{2} \cdot \ldots\left(n_{l}-1\right)=2^{j}$ summed on all values of $l=1, \ldots, j$ is exactly $2^{j}$. On the other hand, given $j, N_{a}=\frac{2^{\hat{h}-j-1}}{n_{a}}$ and $N_{b}=\frac{2^{\hat{h}-j-1}}{n_{b}}$. Hence $n_{a}$ and $n_{b}$ can each have any value $2^{x}$ with $x=0,1, \ldots, \hat{h}-\mathrm{j}-1$. That is, for any given $j$ the number of different combinations of $n_{a}$ and $n_{b}$ is given by $(\hat{h}-j)^{2}$. Overall, by summing upon all $j=1, \ldots, h^{*}-1$, we have that, for $N=2^{h}$, $T(N)=\sum_{j=1}^{\hat{h}-1} 2^{j}(\hat{h}-j)^{2}$. For instance, for $N=4096=2^{13}$, we have $T(N)=\sum_{j=1}^{12} 2^{j}(13-j)^{2}=$ 48756. When $N$ is not a power of 2 , the analysis becomes more tedious while the combinatorial explosion is even larger. Just to give a rough idea on the matter, if we suppose one wants to compute the number of different configurations with $\alpha=0.5, n_{a}=n_{b}=1$ and number of levels $t=\hat{h}$ : then, when the prime factors of $N$ are all identical (as for $N=2^{\hat{h}}$ ), there is just one configuration. On the other hand, when the prime factors of $N$ are all distinct, for any $t$-uple of these different factors assigned to $t$ different levels, there are $t$ ! different assignments of such factors to the levels (namely all possible permutations of a string with length $t$ ) correspondingly inducing $t$ ! different combinations.

Based on these observations, for the chosen value of $N$, the required calculation time for $h \geq 4$ becomes unacceptable, so that in further studies global optimization techniques have to be implemented to maximize strength or other required properties. 


\section{Results}

\subsection{Influence of hierarchy}

First, we consider the case where only one type of fibre is present, i.e. $\alpha=1$, to evaluate the influence of hierarchy only. The mean strength in the non hierarchical case here is $43.1 \mathrm{GPa}$ ). The allowable configurations are evaluated numerically for the chosen $N$ and $h$ parameters by determining all $n_{1}$ and $n_{a}$ values that give rise to integer values for $N_{a}$. The numbering of the configurations is chosen so as to have increasing $n_{l}$ values as first criterion, and increasing $n_{a}$ values as second. Figure 4 illustrates the calculations for the mean strength of the various hierarchical configurations for $h=2$. A quasi-periodic behaviour is found, related to the $n_{l}$ value (as highlighted in Fig.4b). Maximum strength is obtained when maximizing $n_{a}$ values, for given $n_{l}$ values, and the overall maximum strength value is obtained when maximizing both $n_{1}$ and $n_{a}$ (49.7 GPa). This corresponds to hierarchical architectures where bundles constituted of a minimal number of fibres are present, i.e. where minimum stress redistribution occurs in the material in the fracture process. In 9 cases the non-hierarchical mean strength is exceeded, although values are affected by some fluctuations, due to the statistical nature of the simulations.

\subsection{Influence of hierarchy and fibre mixing}

Next, to additionally evaluate the influence of material heterogeneity together with hierarchy, let us consider a "mixed" bundle with $N=3600$ and $\alpha=0.2$. As explained above, the strength of various different hierarchical structures is evaluated for $h=1,2$, 3. It is important to remember that some statistical variability remains in these data, as simulations are based on randomly assigned singlefibre strengths for fibre types $a$ and $b$. Thus, while calculation results do not provide an absolute comparison between different configurations, results are strongly indicative of the relative strength 
classification. Mean strength results for $h=1$ structures are shown in Fig.5. The $x$-axis represents the

considered configurations (again ordered according to increasing $n_{a}$ ), whilst the corresponding strength is plotted on the $y$-axis in $\log$ scale. In this case, the parameters $n_{a}$ and $n_{b}$ are sufficient to define the structure, i.e. the different configurations are obtained by simply changing the number of bundles of fibres of type $a$ and $b$, since only a single level of hierarchy is present. It is found that in this case hierarchy does not favour maximal strength, because the maximum value $(8.71 \mathrm{GPa})$ is obtained for $\left[n_{a}=1, n_{b}=1\right]$ (configuration number 1260 in Fig.5), which corresponds to the nonhierarchical case. However, many other "hierarchical" configurations generate similar strength values, and appear as local maxima in the plot in Fig.5. These favourable configurations are those where the number of parallel fibres is maximized, both for type $a$ and type $b$ fibres, i.e. when $n_{a}$ and $n_{b}$ are small and/or not too dissimilar in value. Conversely, the minima appear for large values of $n_{a}$ or $n_{b}$, especially if one of the two exceeds a limiting value (e.g. $n_{a}$ or $n_{b}>720$ ). This is highlighted more clearly in Fig.6, where strength values are plotted vs. $n_{a}$ and $n_{b}$ in a 3-D plot.

From Figs. 5 and 6 it is apparent that the choice of hierarchical arrangement can lead to a variation in strength of more than an order of magnitude. These observations lead to the consideration that appropriate choice of fibre arrangements can provide the means to obtain tailormade strength properties, starting from the same constituents. No strength improvement is obtained with respect to the non-hierarchical arrangement, proving that higher-level hierarchy is a key factor in this respect.

Results for $h=2$ are shown in Fig.7. Here, the number of available configurations is greater (a total of 4690), as a function of the additional parameter $n_{1}$. For each given $n_{1}$ value, the same quasiperiodic behaviour as in Figs. 4 and 5 is observed as a function of $n_{1}$, with maximum strength values obtained for simultaneous large values of $n_{a}$ and $n_{b}$ and a similar excursion between maxima 
and minima. Thus, these results are consistent with those for $\alpha=1$ (single fibre type) and $h=2$, and

are in contrast with those for $\alpha=0.2$ and $h=1$. This once again proves the importance of hierarchy in determining a qualitatively different behaviour. Here, for increasing $n_{l}$ values, the number of available $\left(n_{a}, n_{b}\right)$ pairs decreases, so that the number of evaluated configurations also decreases and with it the "period" of the oscillations. The number of configurations with a mean strength greater than the non-hierarchical case is 28 , with a maximum value of $10.1 \mathrm{GPa}$, so the introduction of fibre mixing improves the situation with respect to the single-fibre case.

The results obtained with the chosen fibre fraction $\alpha=0.2$ are qualitatively confirmed for different $\alpha$ values. For example, the case $\alpha=0.5$ is considered (Fig.8). Clearly, for this $\alpha$ value, the overall mean strength increases because of the larger fraction of "strong" fibres (type $a$ ), and the mean strength in the non-hierarchical case is $21.8 \mathrm{GPa}$. Again, a quasi-periodic behaviour is obtained in the configuration space, with the best configurations occurring for various $n_{l}$ values when maximizing $n_{a}$ and $n_{b}$ values, which corresponds to the cases of greatest of "local" stress redistributions. Some variability remains, due to the statistical nature of the simulations. A greater number of configurations (66) provide an improvement with respect to the non-hierarchical case, with a maximum mean strength value of $26.4 \mathrm{GPa}$ (a $21 \%$ improvement). Results are compared in Tables 1 and 2 for $h=2$ and $\alpha=0.2$ and $\alpha=0.5$, respectively.

As shown in Fig.9, results are more complicated for $h=3$. There remains an oscillating quasiperiodic behaviour as a function of the hierarchical configuration, with local maxima and minima, as a function of the $n_{1}, n_{2}, n_{a}$ and $n_{b}$ values. As seen in Table 3 , where the 10 most favourable configurations are reported, the maxima in mean strength can be found in configurations where at least one of these numbers is maximized, in particular the $n_{a}$ value, relative to the "strong" type $a$ fibres, so the analysis for $h=2$ is confirmed. Similar to lower-order hierarchies, maximal attained 
strength values exceed the non hierarchical case $(9.1 \mathrm{GPa})$ in 34 cases, i.e. a smaller number of

cases with respect to $h=2$ and $\alpha=0.5$. This seems to indicate that hierarchical structure is all the more effective for greater high-strength fibre content percentage.

To better analyze these qualitative indications, a systematic study for higher hierarchical levels needs to be carried out. For $h>3$, it is not possible to analyze all configurations, due to computational time, but preliminary data (not reported) shows that the overall tendency highlighted at $h=1,2,3$ seems to continue, with a greater span between minimal and maximal strength values, and the more favourable configurations being close to those where the number of parallel fibres at level $h$ is maximized.

\section{Conclusions}

We have presented a systematic procedure to evaluate the influence of heterogeneity and hierarchy in fibre bundle architectures using a Hierarchical Fibre Bundle Model. The first three hierarchical levels have been investigated and a considerable strength variability as a function of hierarchical configuration has been highlighted, with a mean strength improvement of up to $21 \%$ with respect to the non hierarchical case. The hierarchical configurations with better strength characteristics are those favouring the more spatially confined stress redistributions during fracture. The results in this paper are promising for the improvement of the performance of artificial bioinspired architectures and the design of materials with tailor made properties. To derive more specific and quantitative conclusions, in future a wider investigation of the parameter space will be carried out. A greater number of hierarchical levels will be assessed, using global optimization techniques to avoid exceedingly cumbersome calculations and the emphasis will be particularly on metaheuristics based on local improvement techniques (Gendreau and Potvin, 2010). Also, other 
properties will be investigated, including stiffness and energy dissipation, in order to address the

problem of the simultaneous optimization of different material properties (e.g. strength and toughness), which is a commonplace feature in nature but remains to be effectively achieved in artificial materials.

\section{Acknowledgements}

This work is supported by the ERC Ideas Starting grant n. 279985 "BIHSNAM, Bio-inspired Hierarchical Super Nanomaterials", which is gratefully acknowledged. 


\section{References}

Ackbarow, T., Chen, X., Keten, S., Buehler, M.J., 2007. Hierarchies, multiple energy barriers, and robustness govern the fracture mechanics of alpha-helical and beta-sheet protein domains. Proceedings of the National Academy of Sciences of the United States of America 104, 1641016415.

Autumn, K., Liang, Y.A., Hsieh, S.T., Zesch, W., Chan, W.P., Kenny, T.W., Fearing, R., Full, R.J., 2000. Adhesive force of a single gecko foot-hair. Nature 405, 681-685.

Autumn, K., Peattie, A.M., 2002. Mechanisms of adhesion in geckos. Integrative and Comparative Biology 42, 1081-1090.

Bosia, F., Abdalrahman, T., Pugno, N.M., 2012. Investigating the role of hierarchy on the strength of composite materials: evidence of a crucial synergy between hierarchy and material mixing. Nanoscale 4, 1200-1207.

Bosia, F., Buehler, M.J., Pugno, N.M., 2010. Hierarchical simulations for the design of supertough nanofibers inspired by spider silk Physical Review E 82, 056103.

Bosia, F., Pugno, N., Lacidogna, G., Carpinteri, A., 2008. Mesoscopic modeling of Acoustic Emission through an energetic approach. International Journal of Solids and Structures 45, 58565866.

Bratzel, G., Buehler, M.J., 2012. Sequence-structure correlations in silk: Poly-Ala repeat of N. clavipes MaSp1 is naturally optimized at a critical length scale. Journal of the Mechanical Behavior of Biomedical Materials 7, 30-40.

Buehler, M.J., Qin, Z., Cranford, S., Ackbarow, T., 2009. Robustness-Strength Performance of Hierarchical Alpha-Helical Protein Filaments. International Journal of Applied Mechanics 1, 85112. 
Cranford, S.W., Buehler, M.J., 2011. Shaky foundations of hierarchical biological materials. Nano Today 6, 332-338.

Currey, J.D., 1999. The design of mineralised hard tissues for their mechanical functions. Journal of Experimental Biology 202, 3285-3294.

Currey, J.D., 2002. Bones : structure and mechanics. Princeton University Press, Princeton, NJ.

Currey, J.D., 2003. How well are bones designed to resist fracture? Journal of Bone and Mineral Research 18, 591-598.

Espinosa, H.D., Juster, A.L., Latourte, F.J., Loh, O.Y., Gregoire, D., Zavattieri, P.D., 2011. Tabletlevel origin of toughening in abalone shells and translation to synthetic composite materials. Nature Communications 2.

Foelix, R.F., 1996. Biology of spiders, 2nd ed. Oxford University Press ; Georg Thieme Verlag, New York [Stuttgart].

Fratzl, P., Weinkamer, R., 2007. Nature's hierarchical materials. Progress in Materials Science 52, 1263-1334.

Gao, H.J., 2006. Application of fracture mechanics concepts to hierarchical biomechanics of bone and bone-like materials. International Journal of Fracture 138, 101-137.

Gao, H.J., Ji, B.H., 2004. Mechanical properties of nanostructure of biological materials. Journal of the Mechanics and Physics of Solids 52, 1963-1990.

Gendreau, M., Potvin, J.-Y., 2010. Handbook of metaheuristics, 2nd ed. Springer, New York.

Gibson, L.J., Ashby, M.F., Karam, G.N., Wegst, U., Shercliff, H.R., 1995. The MechanicalProperties of Natural Materials .2. Microstructures for Mechanical Efficiency. Proceedings of the Royal Society of London Series a-Mathematical and Physical Sciences 450, 141-162.

Gibson, R.F., 2007. Principles of composite material mechanics, 2nd ed. CRC Press, Boca Raton. 
Keten, S., Xu, Z.P., Ihle, B., Buehler, M.J., 2010. Nanoconfinement controls stiffness, strength and

mechanical toughness of beta-sheet crystals in silk. Nature Materials 9, 359-367.

Lakes, R., 1993. Materials with Structural Hierarchy. Nature 361, 511-515.

Launey, M.E., Buehler, M.J., Ritchie, R.O., 2010. On the Mechanistic Origins of Toughness in Bone. Annual Review of Materials Research, Vol 40 40, 25-53.

Mishnaevsky, L., 2011. Hierarchical composites: Analysis of damage evolution based on fiber bundle model. Composites Science and Technology 71, 450-460.

Nova, A., Keten, S., Pugno, N.M., Redaelli, A., Buehler, M.J., 2010. Molecular and Nanostructural Mechanisms of Deformation, Strength and Toughness of Spider Silk Fibrils. Nano Letters 10, 26262634.

Pradhan, S., Hansen, A., Chakrabarti, B.K., 2010. Failure processes in elastic fiber bundles. Reviews of Modern Physics 82, 499-555.

Pugno, N.M., 2006. Mimicking nacre with super-nanotubes for producing optimized supercomposites. Nanotechnology 17, 5480-5484.

Pugno, N.M., Bosia, F., Carpinteri, A., 2008. Multiscale stochastic simulations for tensile testing of nanotube-based macroscopic cables. Small 4, 1044-1052.

Puxkandl, R., Zizak, I., Paris, O., Keckes, J., Tesch, W., Bernstorff, S., Purslow, P., Fratzl, P., 2002. Viscoelastic properties of collagen: synchrotron radiation investigations and structural model. Philosophical Transactions of the Royal Society of London Series B-Biological Sciences 357, 191197.

Rho, J.Y., Kuhn-Spearing, L., Zioupos, P., 1998. Mechanical properties and the hierarchical structure of bone. Medical Engineering \& Physics 20, 92-102.

Riley, G., 2005 Chronic tendon pathology: molecular basis and therapeutic implications Expert 
Reviews in Molecular Medicine 7, 1-25.

Tian, Y., Pesika, N., Zeng, H.B., Rosenberg, K., Zhao, B.X., McGuiggan, P., Autumn, K., Israelachvili, J., 2006. Adhesion and friction in gecko toe attachment and detachment. Proceedings of the National Academy of Sciences of the United States of America 103, 19320-19325.

Vincent, J.F.V., 1999. From cellulose to cell. Journal of Experimental Biology 202, 3263-3268.

Wegst, U.G.K., Ashby, M.F., 2004. The mechanical efficiency of natural materials. Philosophical Magazine 84, 2167-2181.

Weibull, W., 1939. A statistical theory of the strength of materials. Ingeniörsvetenskapsakademiens Handlingar 151.

Weibull, W., 1951. A statistical distribution function of wide applicability. Journal of Applied Mechanics -Transactions ASME 18, 293-297.

Weiner, S., Wagner, H.D., 1998. The material bone: Structure mechanical function relations. Annual Review of Materials Science 28, 271-298.

Yao, H., Gao, H., 2006. Mechanics of robust and releasable adhesion in biology: Bottom-up designed hierarchical structures of gecko. Journal of the Mechanics and Physics of Solids 54, 11201146. 


\section{List of Figure Captions}

Fig.1: a) Hierarchical structure of tendon (from (Riley, 2005 )), b) Schematization of the hierarchical procedure in multiscale simulations

Fig.2: Two-parameter Weibull distribution $p(\sigma)$ for the strength distribution of level-0 fibres in the FBM: $\sigma_{0}$ is the scale parameter and $m$ is the shape parameter (in this case $m=2$ )

Fig.3: a) Schematization of an example of a 3-level hierarchical structure; b) Schematization of the corresponding reference non-hierarchical structure.

Fig4.: a) Simulations results for the mean strength of different fibre arrangements for $h=2$ and $\alpha=1$; b) close-up on the first 250 configurations, highlighting the quasi-periodic dependency on the $\mathrm{n}_{1}$ index. The dotted line indicates the mean strength value for the non-hierarchical case.

Fig.5: Simulations results for the mean strength of different fibre arrangements for $h=1$ and $\alpha=0.2$. The dotted line indicates the mean strength value for the non-hierarchical case.

Fig.6: 3-D plot of mean strength simulations results for $h=1$ and $\alpha=0.2$

Fig.7: Mean strength simulations results for $h=2$ and $\alpha=0.2$. The dotted line indicates the mean strength value for the non-hierarchical case.

Fig.8: Mean strength simulations results for $h=2$ and $\alpha=0.5$.

Fig.9: Mean strength simulations results for $h=3$ and $\alpha=0.2$

\section{List of Table captions}

Table 1: Examples of configurations providing maxima in mean strength for $h=2$ simulations, with $\alpha=0.2$.

Table 2: Examples of configurations providing local maxima in mean strength for $h=2$ simulations, with $\alpha=0.5$

Table 3: Maxima in mean strength for $h=3$ simulations, with $\alpha=0.2$ 

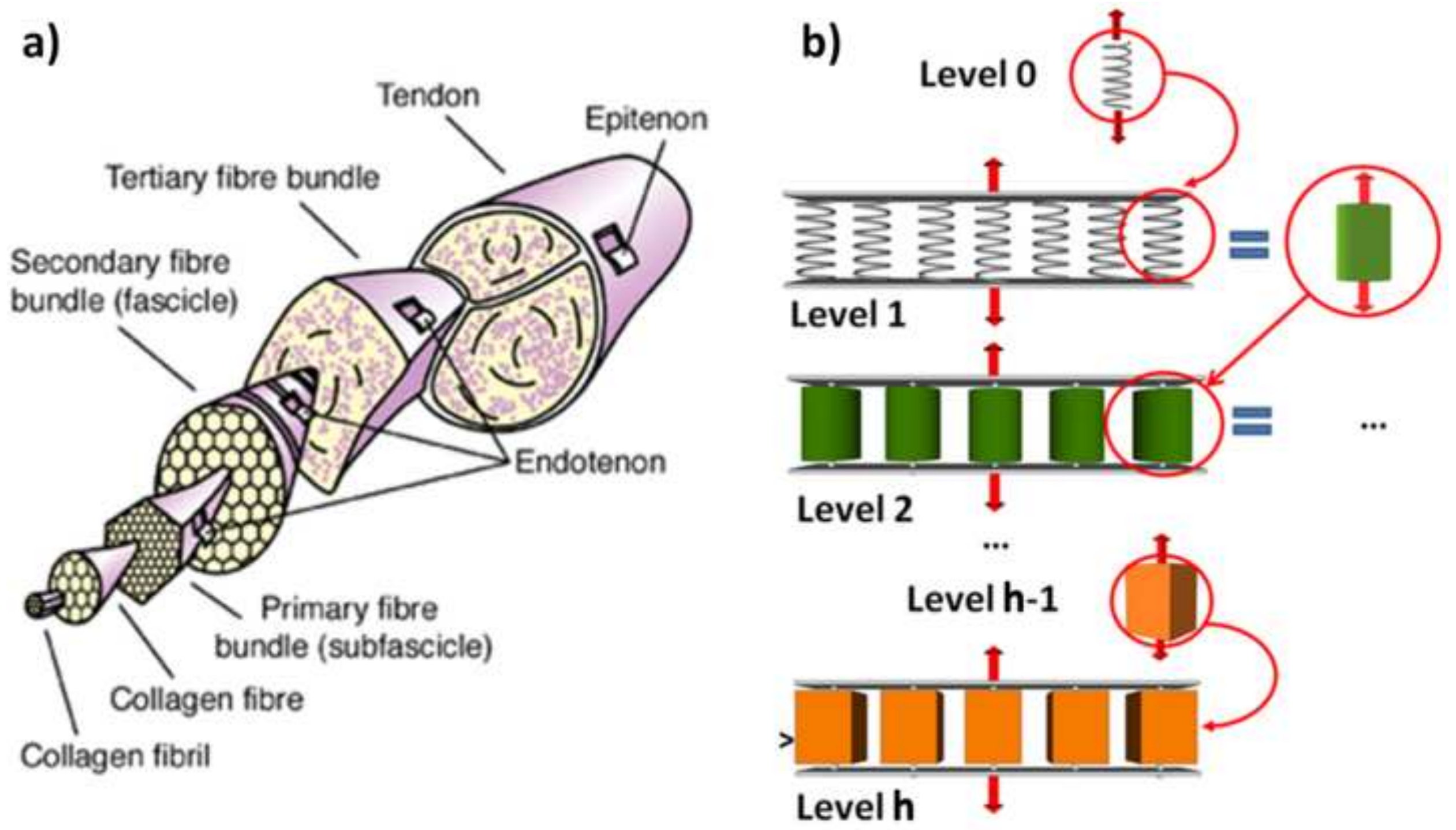
Click here to download high resolution image

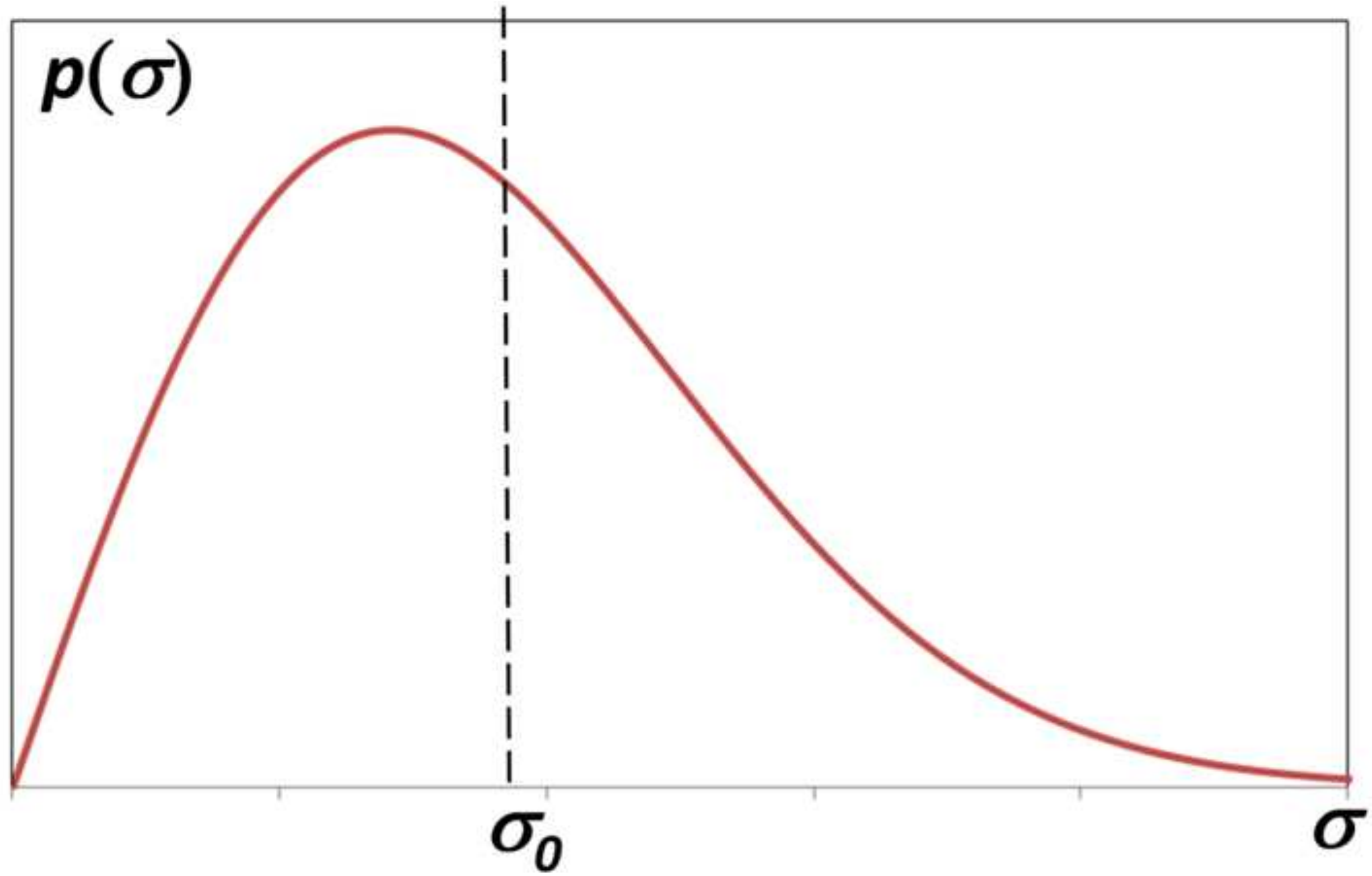




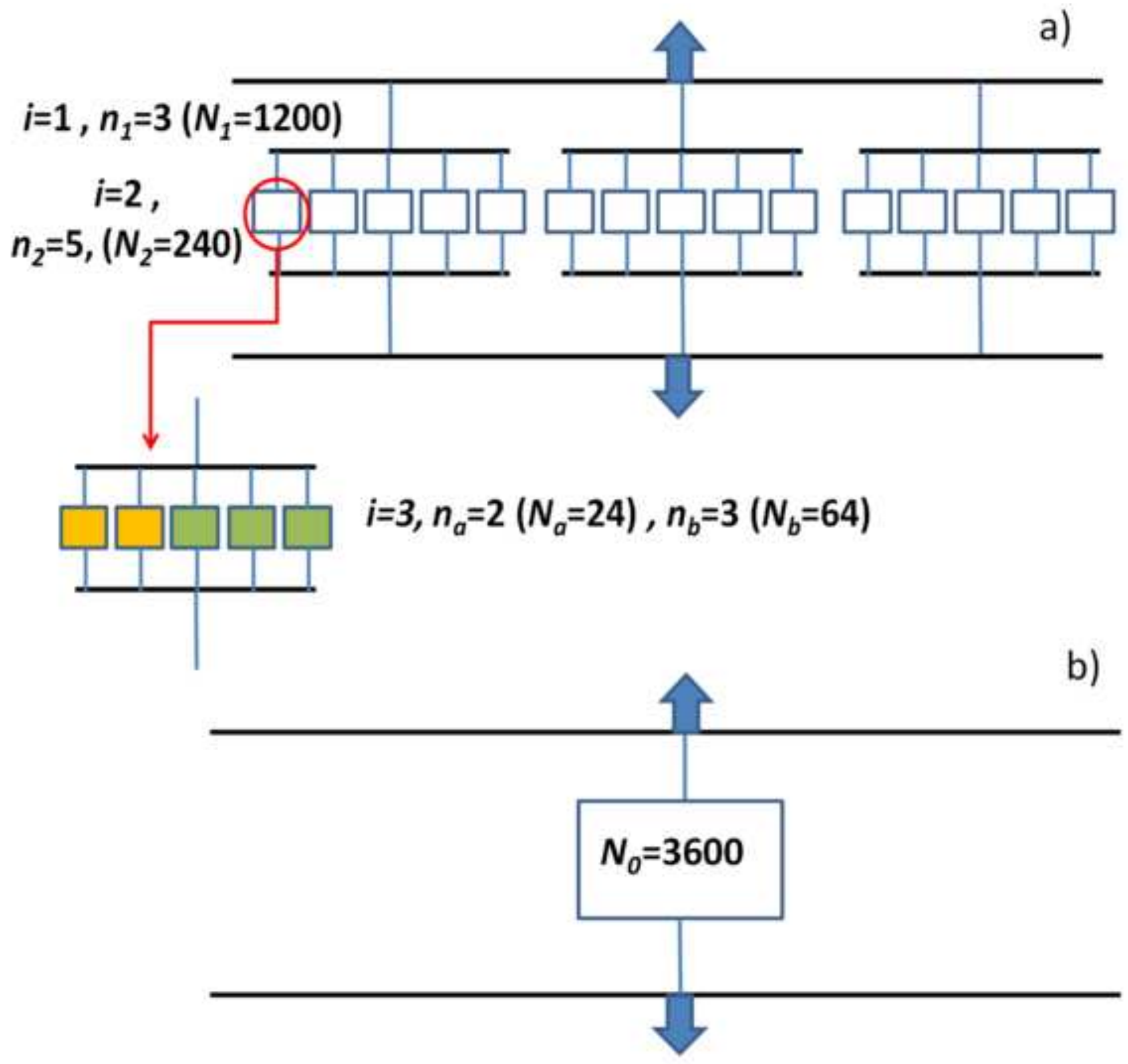



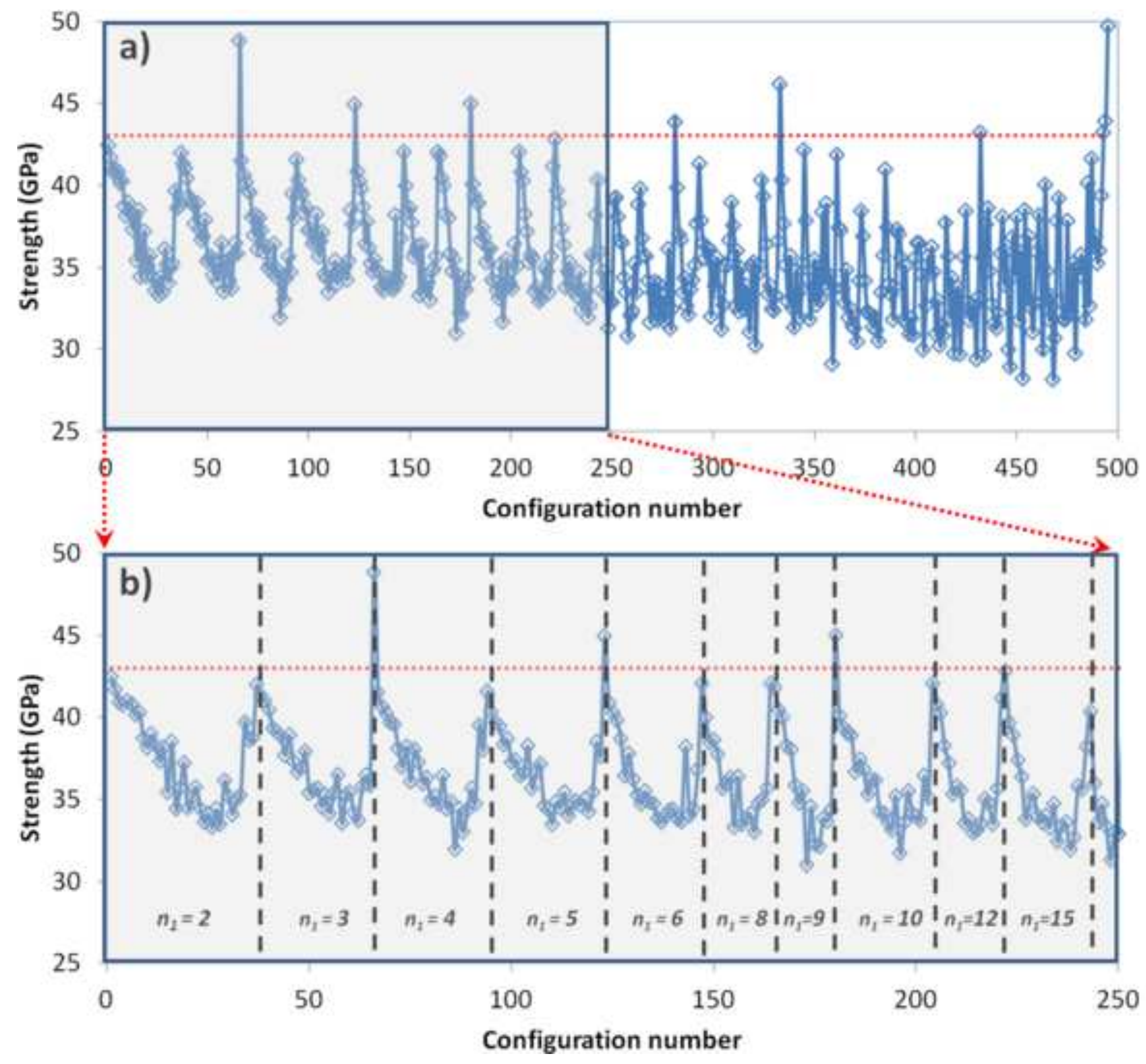


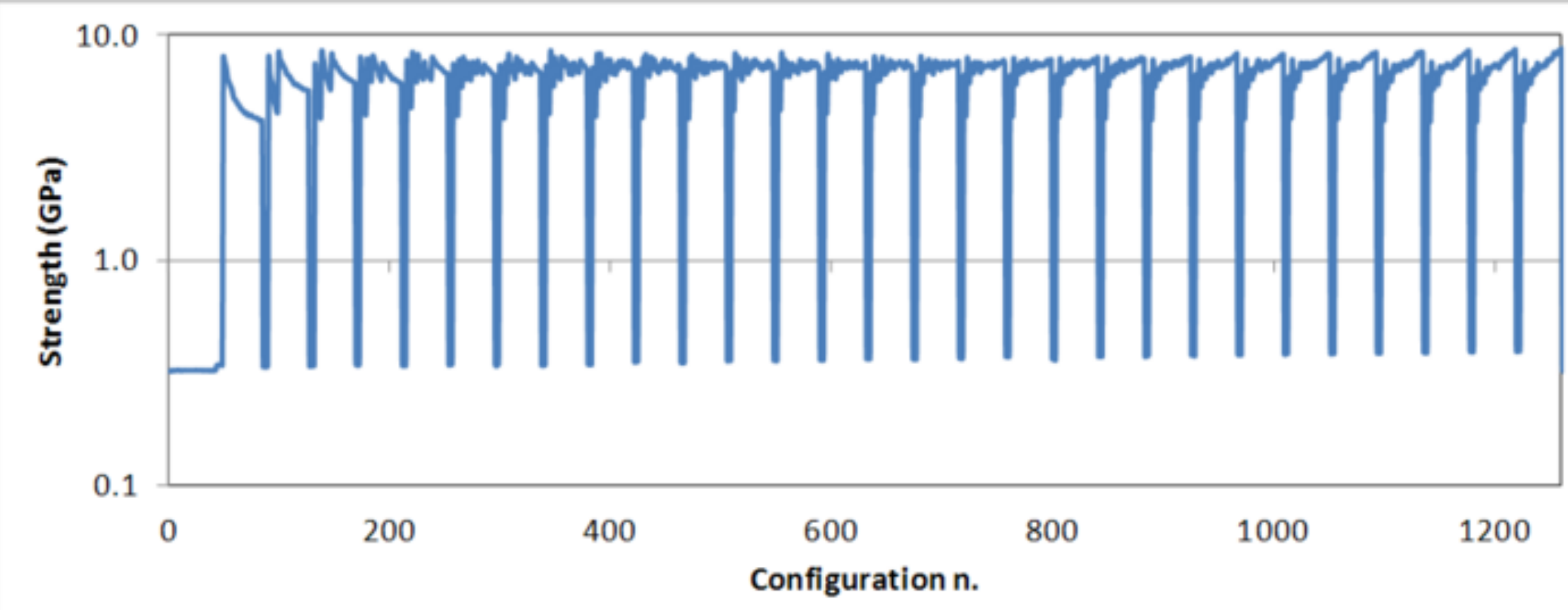




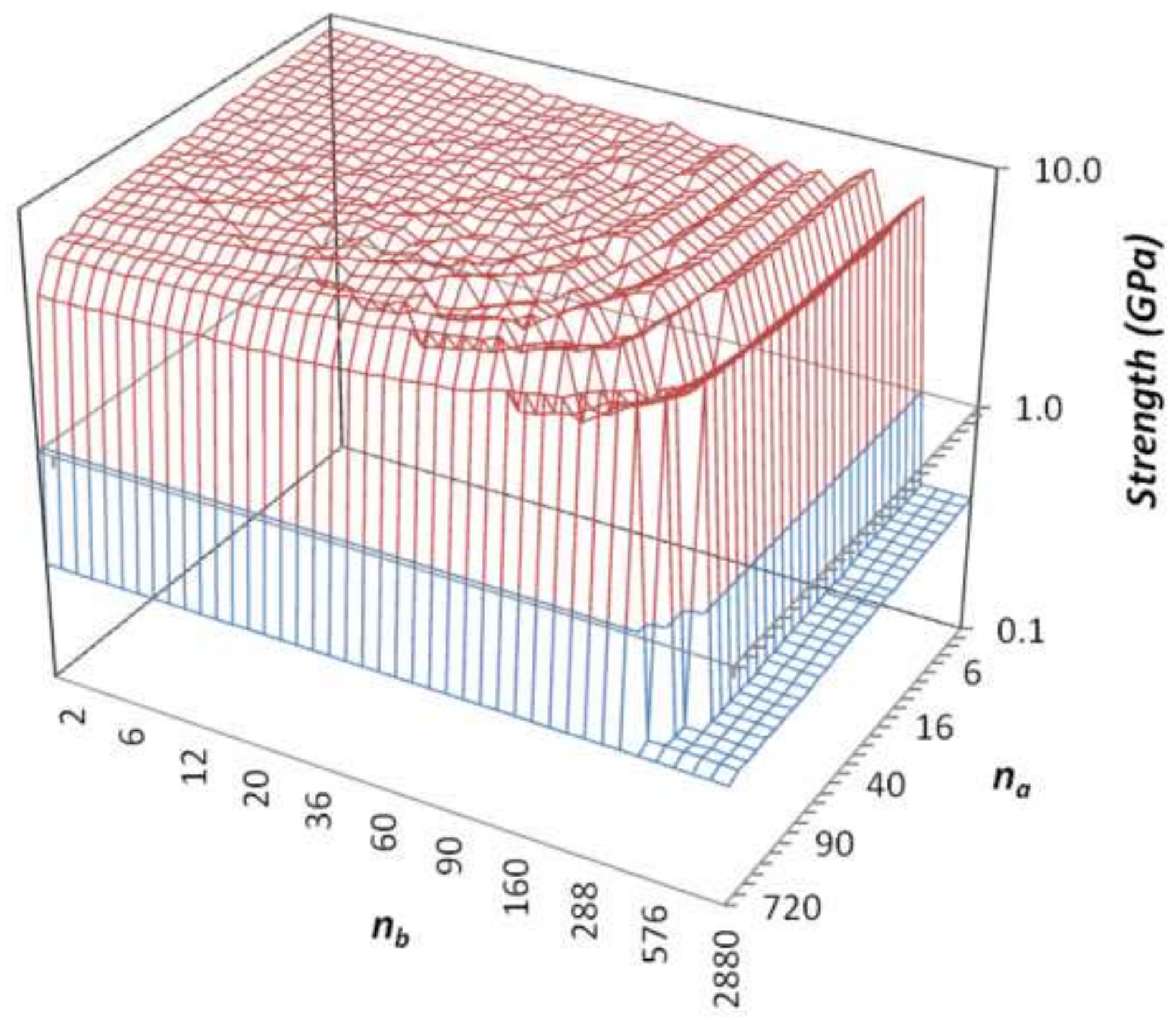




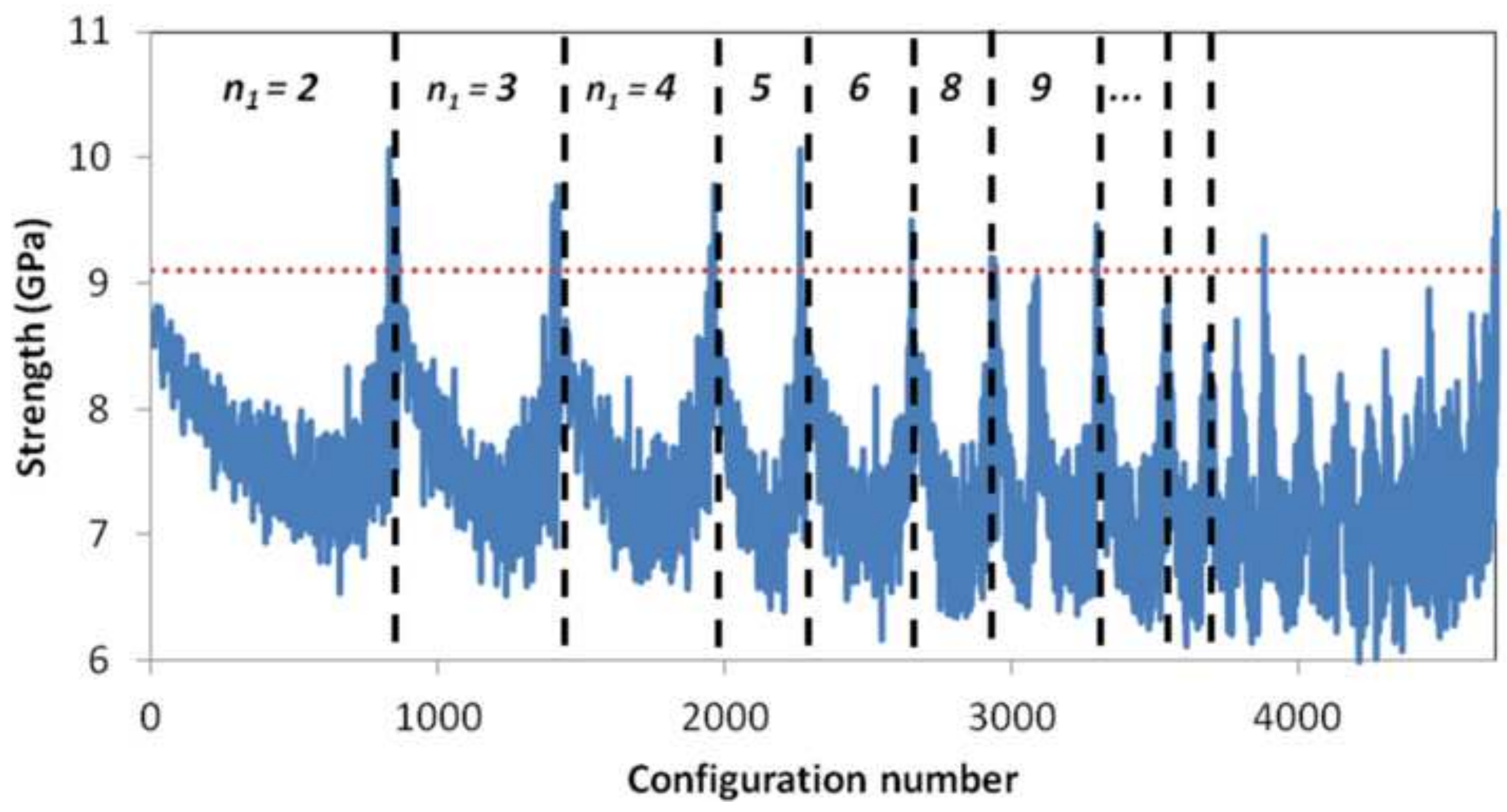




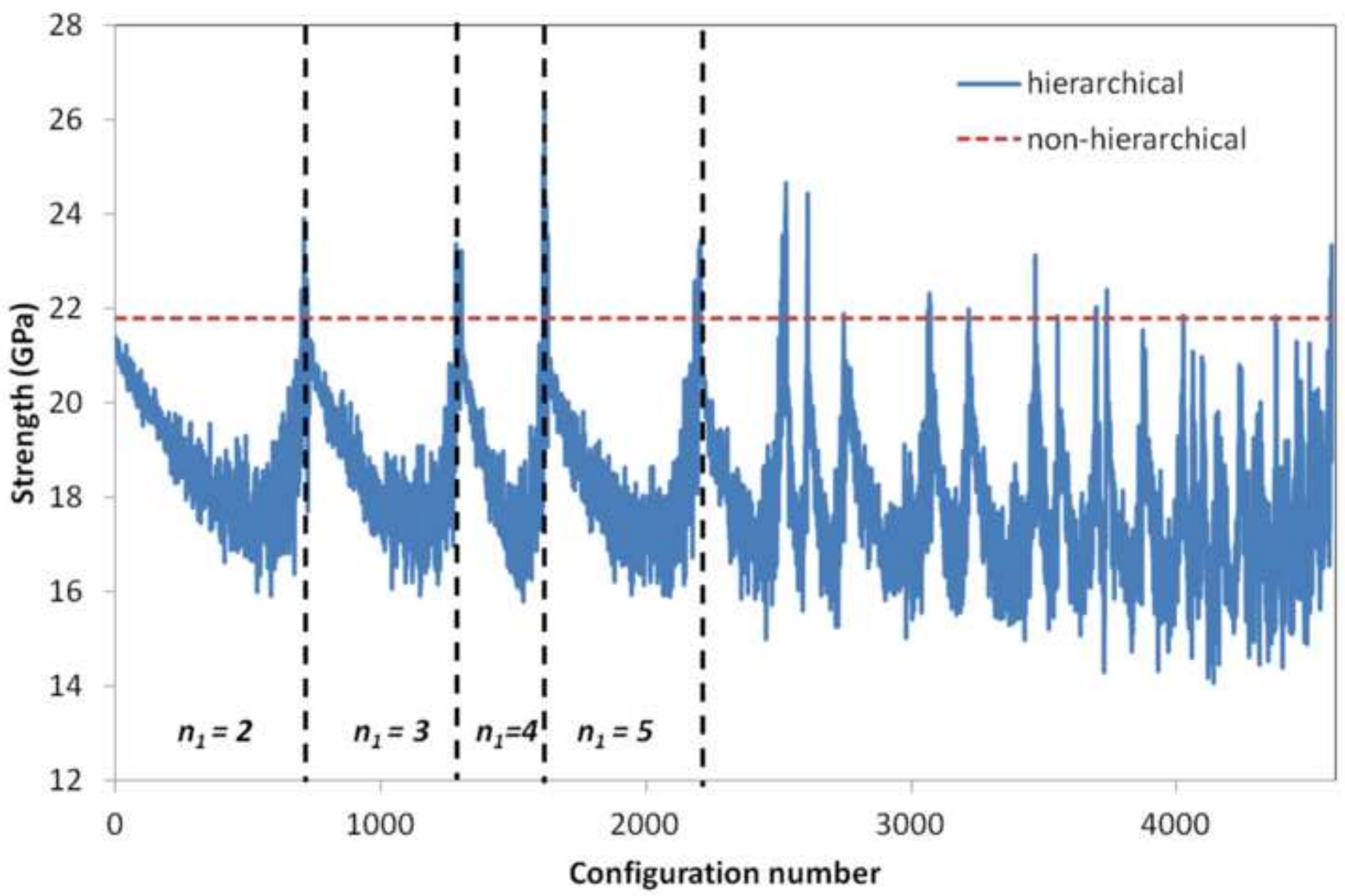




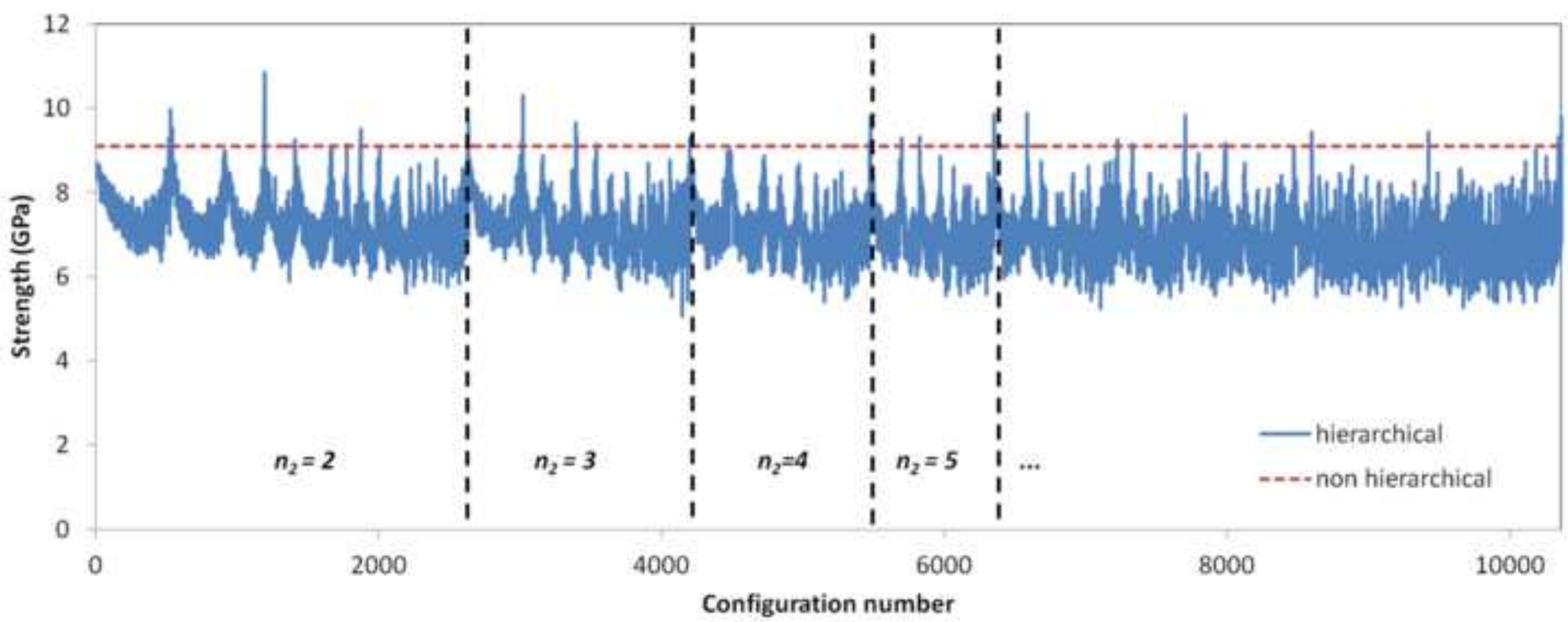


Tables

Table 1

\begin{tabular}{|c|c|c|c|}
\hline$n_{1}$ & $n_{a}$ & $n_{b}$ & Strength $(\mathrm{GPa})$ \\
\hline 5 & 144 & 144 & 10.07 \\
\hline 2 & 360 & 720 & 10.07 \\
\hline 2 & 360 & 20 & 9.81 \\
\hline 3 & 240 & 12 & 9.78 \\
\hline 2 & 360 & 10 & 9.76 \\
\hline 4 & 180 & 2 & 9.75 \\
\hline 3 & 240 & 192 & 9.64 \\
\hline 3 & 240 & 6 & 9.63 \\
\hline 3 & 240 & 64 & 9.57 \\
\hline 720 & 1 & 1 & 9.57 \\
\hline
\end{tabular}


Table 2

\begin{tabular}{|c|c|c|c|}
\hline$n_{1}$ & $n_{a}$ & $n_{b}$ & Mean Strength (GPa) \\
\hline 4 & 450 & 9 & 26.4 \\
\hline 4 & 450 & 2 & 25.2 \\
\hline 6 & 300 & 100 & 24.6 \\
\hline 8 & 225 & 75 & 24.4 \\
\hline 4 & 450 & 45 & 24.2 \\
\hline 6 & 300 & 60 & 24.0 \\
\hline 2 & 900 & 12 & 23.9 \\
\hline 6 & 300 & 300 & 23.6 \\
\hline 6 & 300 & 3 & 23.6 \\
\hline 4 & 450 & 450 & 23.5 \\
\hline
\end{tabular}


Table 3

\begin{tabular}{|c|c|c|c|c|c|}
\hline Configuration n. & $n_{l}$ & $n_{2}$ & $n_{a}$ & $n_{b}$ & Mean Strength (GPa) \\
\hline 1192 & 2 & 4 & 90 & 4 & 10.8 \\
\hline 3020 & 3 & 2 & 120 & 480 & 10.3 \\
\hline 527 & 2 & 2 & 180 & 36 & 10.0 \\
\hline 6582 & 6 & 2 & 60 & 6 & 9.9 \\
\hline 6354 & 5 & 144 & 1 & 1 & 9.9 \\
\hline 7704 & 8 & 90 & 1 & 4 & 9.8 \\
\hline 5480 & 4 & 180 & 1 & 2 & 9.8 \\
\hline 10359 & 360 & 2 & 1 & 2 & 9.8 \\
\hline 3393 & 3 & 4 & 60 & 24 & 9.7 \\
\hline 2634 & 2 & 360 & 1 & 1 & 9.6 \\
\hline
\end{tabular}

